\title{
Induction of Bacteriophage from Members of the Mycobacterium avium, Mycobacterium intracellulare, Mycobacterium scrofulaceum Serocomplex
}

\author{
By TERRY L. TIMME AND PATRICK J. BRENNAN* \\ Department of Microbiology, Colorado State University, Fort Collins, Colorado 80523, USA
}

(Received 7 September 1983; revised 7 March 1984)

\begin{abstract}
Bacteriophages have been induced from strains in the Mycobacterium avium, Mycobacterium intracellulare, Mycobacterium scrofulaceum serocomplex by exposure of cultures to UV light or treatment with mitomycin C. One-sixth of the strains examined, representing all but one of the 31 authenticated serotypes, were found to possess phages lytic for a Mycobacterium smegmatis indicator strain. Four single-plaque isolated phages, TM4, TM9, TM10 and TM20, were purified and shown to have a similar morphology on electron micrographs. They had an isometric head of diameter $50-58 \mathrm{~nm}$ and a flexible non-contractile tail about $170 \mathrm{~nm}$ in length with a terminal bulb. All had an identical buoyant density in $\mathrm{CsCl}$ of $1.521 \mathrm{~g} \mathrm{~cm}^{-3}$ and extreme sensitivity to chloroform. The induced phages differed in host range and possessed the unique ability to lyse other members of the serocomplex. Interest in these phages centres on a possible role in mediating genetic interrelationships between members of the serocomplex.
\end{abstract}

\section{INTRODUCTION}

Strains of Mycobacterium avium, Mycobacterium intracellulare and Mycobacterium scrofulaceum are grouped together as a biochemically related but serologically distinct complex, termed the MAIS serocomplex (Wolinsky \& Schaefer, 1973). These organisms are widespread in nature, and, despite their relatively low virulence, are among the most common of the so-called 'atypical' mycobacteria associated with disease (Wolinsky, 1979). One of the most distinctive features of MAIS organisms is an outer filamentous layer or capsule composed of a unique group of glycopeptidolipids generically known as 'C-mycosides' (Draper \& Rees, 1973; Draper, 1974, 1982; Barrow et al., 1980). Serological specificity among members of the MAIS complex is due to variations in the oligosaccharide components of the more polar glycopeptidolipids (Brennan \& Goren, 1979; Brennan et al., 1981). The singly glycosylated apolar glycopeptidolipids can serve as the receptor for phage D4 in Mycobacterium smegmatis and in an unidentified Mycobacterium species designated 1217 (Furuchi \& Tokunaga, 1972; Goren et al., 1972). The apolar C-mycoside from $M$. scrofulaceum was capable of inactivating phage D4 to the same extent as that from the other two mycobacteria; however the $M$. scrofulaceum cells did not adsorb the phage and were resistant to lysis by D4 phage (Goren et al., 1972). Indeed, the resistance of members of the MAIS serocomplex to lysis by numerous phages is well documented (Takeya et al., 1959; Buraczewska et al., 1971; Crawford et al., 1981). One possible reason for resistance could be the presence of resident prophages inducing a state of superinfection immunity.

Lysogeny and pseudolysogeny are quite common in the genus Mycobacterium and the subject of recent reviews (Rado \& Bates, 1980; Grange, 1982). Pseudolysogeny is the term applied to a non-integrated phage carrier state characterized by the spontaneous liberation of phage particles and the segregation of phage-sensitive bacteria (Baess, 1970). Lysogenic bacteria are immune to homologous phage and usually can be induced to produce phages by treatment of the culture with agents which inactivate the phage repressor, such as UV light or mitomycin C. Previous attempts to induce bacteriophage from $M$. avium with UV light have generally been

Abbreviation: MAIS, Mycobacterium avium/Mycobacterium intracellulare/Mycobacterium scrofulaceum. 
unsuccessful (Russell et al., 1960), though Buraczewska et al. (1971) stated that they had been able to induce phage from 'atypical' mycobacteria by UV irradiation. However, evidence of strain characterization was not presented and the phages induced were only able to lyse saprophytic mycobacteria and some $\boldsymbol{M}$. tuberculosis strains.

The purpose of this investigation was to examine members of the MAIS serocomplex for the presence of inducible bacteriophages to probe the genetic interrelationship of strains in the MAIS complex. This report describes the methods used to induce phages and presents a characterization of four phages which are capable of lysing members of the MAIS serocomplex.

\section{METHODS}

Bacteria. All of the strains of $\boldsymbol{M}$. acium, $\boldsymbol{M}$. intracellulare and $\boldsymbol{M}$. scrofulaceum obtained from National Jewish Hospital and Research Center, Denver, Colorado, USA are listed in Table 1. Most are part of an authenticated culture collection (Tsang et al., 1983). The serotype designation of all the MAIS strains was confirmed by TLC of the alkali-stable type-specific polar glycopeptidolipids (Brennan et al., 1982) and by seroagglutination (Tsang $e t$ al. . 1983). All cultures were started from single colonies which had smooth, opaque, colonial morphology. Strain 607 of M. smegmatis (American Type Culture Collection) was used as the indicator organism and for phage propagation.

Media and solutions. Bacteria were routinely grown in $7 \mathrm{H} 11$ broth (Cohn et al., 1968) containing 0.05\% $(\mathrm{v} / \mathrm{v})$ Tween 80 . Cells were washed and diluted in $0.85 \%(\mathrm{v} / \mathrm{v})$ saline containing $0.05 \%(\mathrm{v} / \mathrm{v})$ Tween 80 . Brain heart infusion broth containing $2 \mathrm{mM}-\mathrm{CaCl}_{2}$ was used for mitomycin $\mathrm{C}$ induction and as phage diluent. Tris/ $\mathrm{HCl}$ ( $50 \mathrm{mM}, \mathrm{pH} 9.0$ ) was also used as a phage diluent and buffer. The soft agar overlay technique was used for all phage assays with supplemented Dubois broth base without Tween 80 as the soft agar (Jones \& Greenberg, 1978), and 7H1l agar as the hard basal medium.

Phage induction and purification. Exponential phase cultures were diluted twofold in $7 \mathrm{H} 11$ broth and incubated at $37^{\circ} \mathrm{C}$ with agitation for $36 \mathrm{~h}$. They were centrifuged $(4000 \mathrm{~g}, 10 \mathrm{~min})$, washed once, and resuspended in onetenth the original volume of saline. The concentrated cell suspension was divided into two aliquots. One was diluted to the original volume with saline and irradiated for $1.5 \mathrm{~min}$ with UV light with a maximum emission at $254 \mathrm{~nm}$ and flux of $1.05 \mathrm{~J} \mathrm{~m}^{-2} \mathrm{~s}^{-1}$. After UV exposure the cells were centrifuged and then resuspended in fresh $7 \mathrm{H} 11$ broth. The cells for mitomycin $\mathrm{C}$ induction were added to the original volume of $\mathrm{CaCl}_{2}$-containing brain heart infusion broth which contained $2.5 \mu \mathrm{g}$ mitomycin $\mathrm{C} \mathrm{ml}{ }^{-1}$ (Sigma). Cells from both types of induction were incubated in the dark at $37^{\circ} \mathrm{C}$ for $24 \mathrm{~h}$. They were then centrifuged and the supernatant filtered through a $0 \cdot 2 \mu \mathrm{m}$ pore size filter. Soft agar overlays of the indicator bacteria were spotted with $20 \mu$ of the filtrate or a dilution thereof. The plates were incubated at $37^{\circ} \mathrm{C}$ and examined at 24 and $48 \mathrm{~h}$. When well isolated plaques appeared they were picked with a sterile needle and resuspended in a small volume of a fresh broth culture of the indicator organism. After overnight incubation this was centrifuged and the supernatant filtered as above. Serial tenfold dilutions were spotted onto soft agar overlays of the indicator. A well isolated single plaque was again picked, mixed with fresh indicator cells and incubated overnight. The phage suspension was centrifuged and filtered prior to titration. Dilutions of the phage suspension were used to prepare high titre stocks by harvesting soft agar overlays which displayed near confluent lysis.

In some cases high titre phage lysates were purified by banding in $\mathrm{CsCl}$ block gradients. The phage suspension was made $10 \mathrm{mM}$ with respect to $\mathrm{MgSO}_{4}$ and treated for $2 \mathrm{~h}$ at $37^{\circ} \mathrm{C}$ with DNAase I and RNAase A (Sigma) at $50 \mu \mathrm{g} \mathrm{ml} l^{-1}$. After centrifugation $(10000 \mathrm{~g}, 15 \mathrm{~min})$, the supernatant was dialysed against Tris buffer. A $20 \mathrm{ml}$ sample of the dialysed phage suspension was placed in a nitrocellulose tube and underlaid with $2.0 \mathrm{ml} 20 \%(\mathrm{w} / \mathrm{v})$ sucrose and three bands of $\mathrm{CsCl}$ at densities of $1.300,1.500$ and $1.700 \mathrm{~g} \mathrm{ml}^{-1}$. The tubes were centrifuged in a SW 27 rotor at 25000 r.p.m. for $4 \mathrm{~h}$ at $4^{\circ} \mathrm{C}$ and the opalescent phage band collected through the side of the tube and dialysed.

Host range. Strains of mycobacteria were prepared as soft agar overlays and spotted with $20 \mu l$ drops of three dilutions $(1000 \times, 10 \times$ and $I \times)$ of phage. The concentration of phage was adjusted so that the greatest dilution $(1 \times)$ gave $10-20$ plaques on $M$. smegmatis overlays.

Electron microscopy. High titre phage lysates were prepared for electron microscopy by centrifugation at $100000 \mathrm{~g}$ for $1 \mathrm{~h}$. The pellet was resuspended in one-hundredth the volume of brain heart infusion broth. The phages were allowed to adsorb to carbon-reinforced, Formvar-coated grids and negatively stained with $1 \cdot 5 \%(\mathrm{w} / \mathrm{v})$

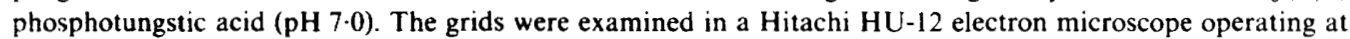
$75 \mathrm{kV}$.

Phage buoyant density: About $8 \mathrm{ml}$ phage lysate was placed in a nitrocellulose tube. Dry $\mathrm{CsCl}$ was added until the density was $1.500 \mathrm{~g} \mathrm{ml}^{-1}$. The contents were mixed and overlaid with a small volume of $40 \%(\mathrm{w} / \mathrm{w})$ sucrose. Tubes were centrifuged in a SW 40 rotor at 23000 r.p.m. for $40 \mathrm{~h}$ at $18{ }^{\circ} \mathrm{C}$. The obvious phage band was recovered by 
Table 1. MAIS serotypes and strains used for phage induction and host range

\begin{tabular}{|c|c|c|c|c|c|}
\hline Serotype & Strain & Inducer* & Serotype & Strain & Inducer* \\
\hline 1 & $11907-300$ & & 16 & ATCC 15987 & \\
\hline 2 & $14141-1395$ & & 17 & p-54 & UV \\
\hline 3 & 128 Germany & & 18 & Melnick & \\
\hline 4 & $135-1079$ & & 18 & Altmann 4990 & \\
\hline 4 & $6 / 8$ & MC & 19 & Darden & \\
\hline 5 & $25546-759$ & & 19 & $7-15$ & \\
\hline 6 & 34540 Wales & & 20 & TMC 1419 & \\
\hline 7 & $p-49$ & & 20 & NQ & UV \\
\hline 8 & SJB \#2 & & 21 & 2993 & \\
\hline 8 & $9 / 78 / 77$ & & 22 & 10409 & \\
\hline 9 & $17484-286$ & & 23 & CDC 1214 & \\
\hline 9 & Watson & $\mathrm{MC}$ & 24 & 12645 & \\
\hline 10 & $1602-1965$ & UV & 25 & $72-888$ & \\
\hline 10 & TMC 1461 & & 26 & McKenzie & \\
\hline 11 & $14186-1424$ & & 27 & Lane 3081 & \\
\hline 11 & TMC 1462 & & 41 & 2729 Cardiff & \\
\hline 12 & Woodduck & UV & 41 & Bridge & UV \\
\hline 13 & TMC 1466 & & 42 & EW 10407 & \\
\hline 14 & p-39 & & 42 & CDC 1198 & \\
\hline 15 & Simpson & & 43 & Brooks & \\
\hline 16 & Yandle-Yandle & & 43 & $7-16$ & \\
\hline
\end{tabular}

aspiration and the refractive index measured on an American Optical Refractometer. The phage buoyant density was calculated using the formula of Brunner \& Vinograd (1965).

Phage inactivation with organic solvents. The sensitivity of the various phage preparations to organic solvents was determined with slight modifications of previously described procedures (Bradley \& Rutherford, 1975). Bacteriophages were diluted in brain heart infusion broth to a titre of about $2.0 \times 10^{7}$ p.f.u. $\mathrm{ml}^{-1}$ then mixed with redistilled solvent at a lysate : solvent ratio of $3: 1$. The mixture was gently agitated for $4 \mathrm{~h}$ at $37^{\circ} \mathrm{C}$ then briefly centrifuged. The aqueous phase from the biphasic mixture was titrated in triplicate. The control consisted of three parts lysate and one part brain heart infusion broth incubated and titrated in like fashion.

\section{RESULTS AND DISCUSSION}

Of 30 of the 31 authenticated MAIS serotypes only 7 yielded phages detectable with the $M$. smegmatis indicator strain (Table 1). The UV light exposure generally reduced bacterial viability by $50-90 \%$ and was effective at inducing phages capable of lysing the indicator in 5 of the 42 strains tested. Mitomycin $\mathrm{C}$ treatment induced phages lytic for $M$. smegmatis in two of the strains (Table 1). However, the strains with UV light-inducible phage did not liberate phage when treated with mitomycin $\mathrm{C}$. Conversely, the strains with mitomycin $\mathrm{C}$-inducible phage did not yield phage upon exposure to UV light.

The plaques which developed on the indicator were approximately $2 \mathrm{~mm}$ in diameter and turbid. The number of phages detected after induction was typically about $10^{4}$ p.f.u. $\mathrm{ml}^{-1}$. Plaque formation in the mitomycin $\mathrm{C}$-induced strains was frequently obscured by the presence of a bacteriocin-like clearing, a not unexpected event in view of the presence of bacteriocins in a wide variety of mycobacterial species (Takeya \& Tokiwa, 1972). Bacteriocin-like clearing was only observed with undiluted filtrates. Single plaques which developed on the indicator lawn when spotted with filtrate from four different induced cultures were further purified by singleplaque re-isolation. The phages obtained by treatment of strains 6/8 (serotype 4) and Watson (serotype 9) with mitomycin C were designated TM4 and TM9, respectively. Phage TM10 was single-plaque isolated from a UV-induced culture of strain 1602-1965 (serotype 10). Strain NQ (serotype 20) yielded phage designated TM20 upon UV induction. All of the phages were grown to a titre of at least $10^{10}$ p.f.u. $\mathrm{ml}^{-1}$ on $M$. smegmatis.

Phages from the present study were not inactivated by treatment with DNAase or RNAase. $\mathrm{CsCl}$ block gradient purification resulted in a decrease in the phage titre of up to 100 -fold. All of the phage preparations used for the block gradient purification gave rise to several bands, but 


\section{Table 2. Lysis of MAIS strains by UV-and mitomycin C-induced phage}

Single-plaque purified TM4, TM9, TM10 and TM20 were used. Test strains were prepared as soft overlays and spotted with $20 \mu \mathrm{l}$ of $1000 \times, 10 \times$ and $\mathrm{I} \times$ dilutions of phage. Concentrations of each phage preparation were adjusted so that the greatest dilution $(1 \times)$ gave 10-20 plaques on $M$. smegmatis overlays.

Lysis by phage:

\begin{tabular}{cl} 
Serotype & \multicolumn{1}{c}{ Strain } \\
3 & 128-Germany \\
4 & $6 / 8$ \\
7 & p-49 \\
17 & p-54 \\
18 & Melnick \\
19 & Darden \\
25 & $72-888$ \\
41 & 2719 Cardiff \\
42 & EW 10407
\end{tabular}

$\begin{array}{cccc}\text { TM4 } & \text { TM9 } & \text { TM10 } & \text { TM20 } \\ + & + & + & + \\ + & + & + & + \\ + & + & + & + \\ + & - & - & + \\ + & - & + & + \\ + & + & + & + \\ + & + & + & + \\ + & + & + & - \\ + & + & + & +\end{array}$

biologically active phage was located in the lower band. The purified phages were tested for their ability to lyse mycobacteria in the MAIS serocomplex by spot testing. The majority of strains were not lysed by any of the phages (Table 2). About one-fourth of the MAIS strains were sensitive to at least two of the phage isolates and none was sensitive to only one phage. The phages had similar but not identical host range. The four phages could be differentiated by their lytic activity on strains P-54, Melnick and 2729 Cardiff. Phage TM4 lysed all three strains whereas TM9 lysed only one. Phages TM10 and TM20 had non-identical patterns of lysis on two of the three strains. The efficiency of plating of the phage was the same on all strains. There was no apparent correlation of phage sensitivity with serotype and where several strains of a particular serotype were tested they had differing phage sensitivities. There was no indication of restriction of phage growth on any sensitive strain.

Although these phages can be differentiated on the basis of the means of induction and the host range, they are probably derived from a common parent phage, since in $M$. smegmatis lysogenization with either TM4 or TM10 confers immunity to both phages but not to phage D29 (data not presented). Although the phages do not have a wide host range, they are capable of lysing some of the strains from the MAIS serocomplex which were resistant to lysis with the phage used by Crawford et al. (1981). We have not yet tested the phage isolates for ability to lyse mycobacteria outside the MAIS complex, so it is possible that some may prove useful in other phage-typing schemes. Such has been the case with a phage from a scotochromogenic acid-fast bacillus termed 33D which could lyse $\boldsymbol{M}$. tuberculosis and $\boldsymbol{M}$. bovis but not $\boldsymbol{M}$. tuberculosis BCG (Buraczewska et al., 1971). All of the phages used in a preliminary attempt to develop a phagetyping scheme for the MAIS serocomplex (Crawford et al., 1981) and most of the phages used for typing $M$. tuberculosis have been isolated from environmental sources. The lysogenic phage induced from members of the MAIS serocomplex could therefore prove useful for phage-typing purposes.

Electron microscopic examination of negatively stained preparations of the phage revealed that all isolates possessed isometric heads and tails (Fig. 1). The head diameter, measured between apices, was $50-58 \mathrm{~nm}$. Each phage had a tail length of about $170 \mathrm{~nm}$. None of the phages had visible base plates or fibres on the tails, but a small bulbous structure was apparent at the distal end of the tails. This identical morphology is not unexpected since the majority of mycobacteriophages described to date have a similar appearance (Rado \& Bates, 1980) with the exception of the phages induced from 'atypical' mycobacteria with UV light by Buraczewska et al. (1972), which were found to have an elongated head. There was no morphological evidence of phage lipids in any of the phages described herein.

The four TM phages had the same buoyant densities of $1.521 \mathrm{~g} \mathrm{~cm}^{-3}$. The refractive indices of the four preparations varied by less than 0.0002 .

The solvent sensitivities of the phages were similar; they were all extremely sensitive to chloroform with an overall decrease in titre of five orders of magnitude. Methanol reduced the 
(a)
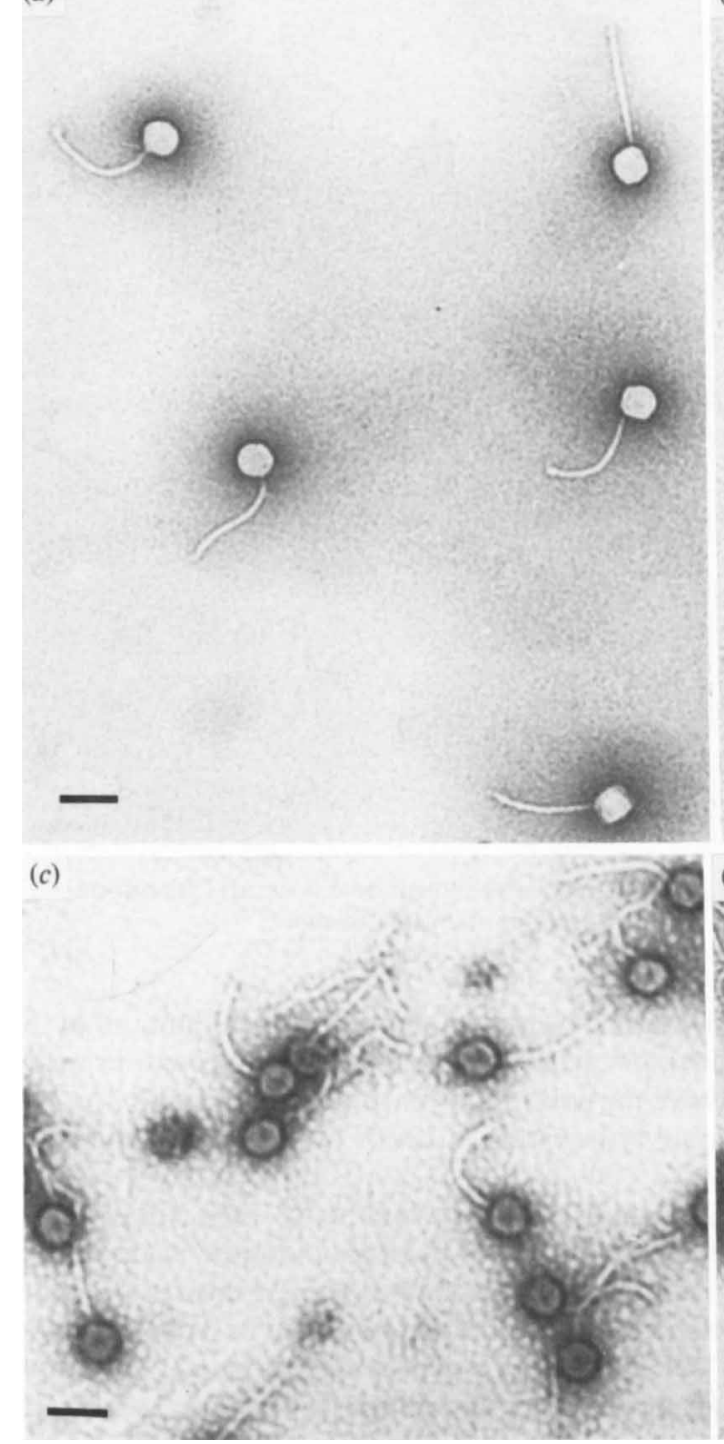

(b)

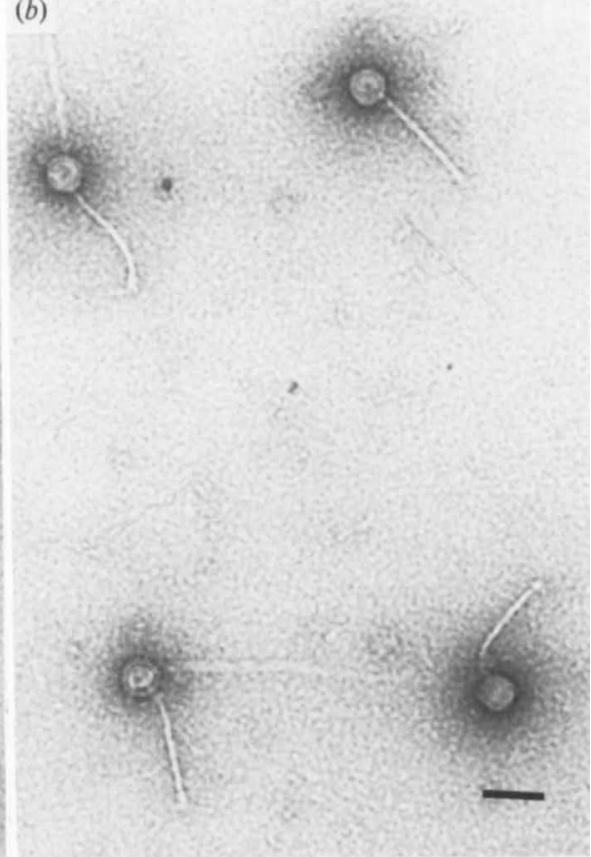

(d)

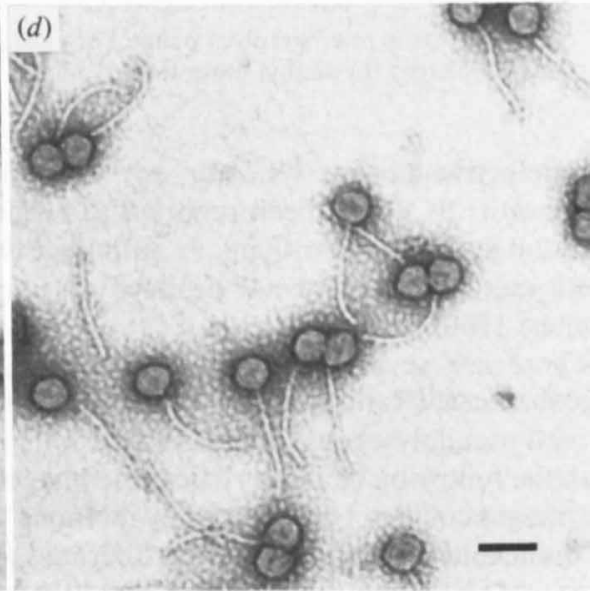

Fig. 1. Electron micrograph of negatively stained phages induced from MAIS strains, and single-plaque purified. (a) Phage TM4, induced with mitomycin C from serotype 4 (strain 6/8); (b) phage TM9 induced with mitomycin $C$ from serotype 9 (strain Watson); (c) phage TM10, UV-induced from serotype 10 (strain 1602-1965); (d) phage TM20, UV-induced from serotype 20 (strain NQ). The bar markers indicate $100 \mathrm{~nm}$.

titre by $10^{-2}$. Diethyl ether had a variable effect, reducing the titre by $10^{-1}$ to $10^{-3}$, but not in a reproducible manner. Electron microscopy of the chloroform- and diethyl ether-treated phages showed no obvious change in morphology. Ether seemed to generate more empty-headed phage particles and, with both TM4 and TM9, there were clusters of phages attached by their tails to an amorphous substance (Fig. 2). The lipid solvent sensitivity of mycobacteriophages is widely recognized yet the location of the lipids or their precise function is unknown (Rado \& Bates, 1982).

To our knowledge, this is the first report of the induction of phages from the MAIS serocomplex capable of lysing other MAIS strains, and of the induction with mitomycin $\mathrm{C}$ of 


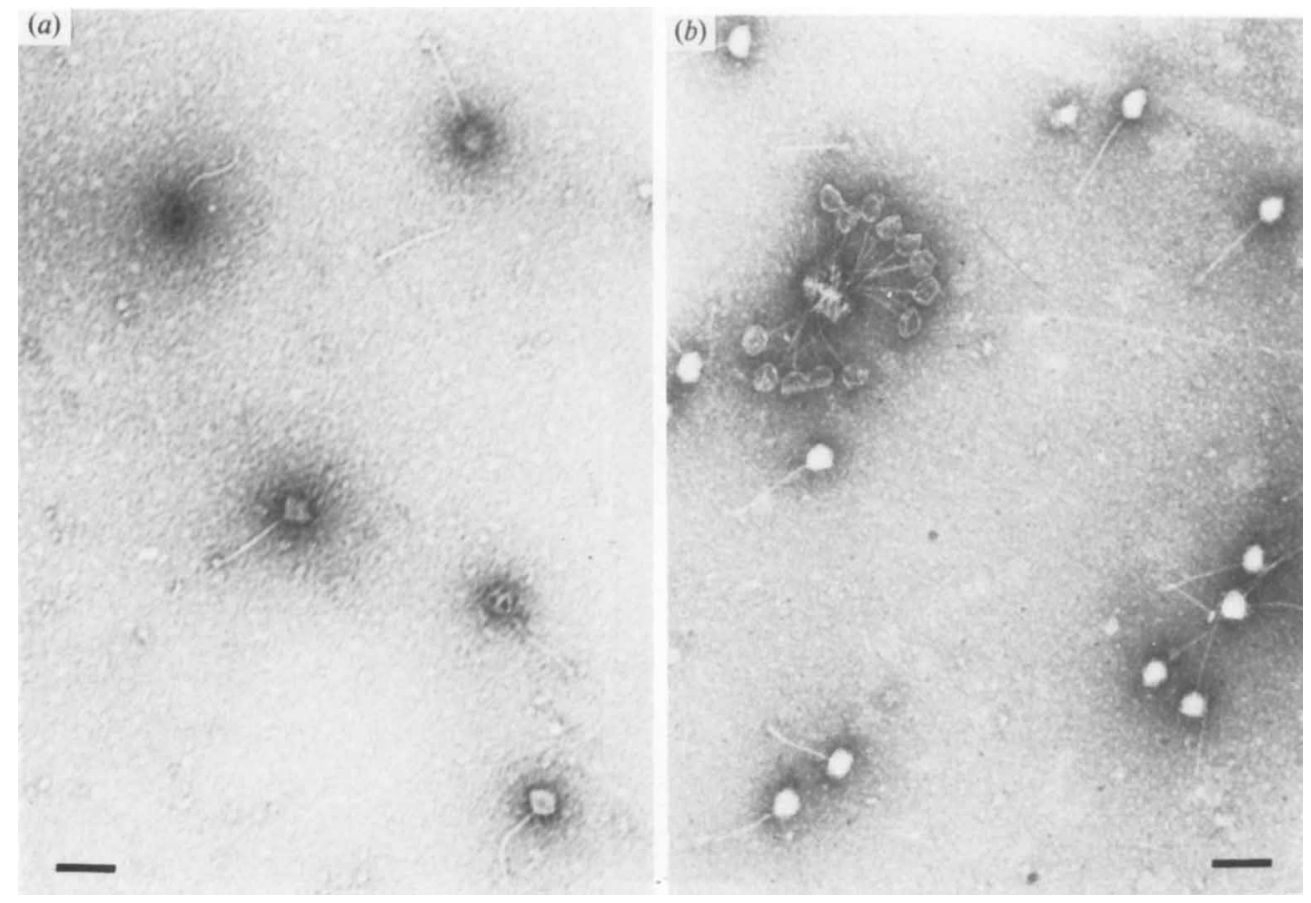

Fig. 2. Electron micrograph of phage TM4 negatively stained after solvent treatment. (a) Chloroformtreated phage; $(b)$ diethyl ether-treated phage. The bar markers indicate $100 \mathrm{~nm}$.

non-defective phages lytic for any mycobacterial species. A morphological mutant of $M$. tuberculosis BCG has been reported to produce phage-like particles after $2 \mathrm{~d}$ growth in media with $2.0 \mu \mathrm{g}$ mitomycin $\mathrm{C} \mathrm{ml}^{-1}$. Although these particles did reduce the number of c.f.u. in a broth culture, they did not produce detectable lysis either in broth or on plates (Imaeda \& Rieber, 1968).

There are several reports of naturally occurring and experimentally induced lysogenic mycobacteria (Rado \& Bates, 1980; Grange, 1982). However, most should more accurately be termed pseudolysogens. Baess (1970) applied this term to mycobacteriophage-host interactions with the following characteristics: the phage DNA did not integrate into the host chromosome; the phages could not be induced by methods which normally induce lysogenic phage; the culture medium contained spontaneously liberated phage particles; and phage-free bacterial segregants appeared in the culture, particularly in the presence of anti-phage serum. Attempts to produce lysogenic or pseudolysogenic $\boldsymbol{M}$. avium or $\boldsymbol{M}$. intracellulare experimentally have been unsuccessful (Russell et al., 1960). These same strains also failed to yield phage upon exposure to UV light. There is but one brief report (Buraczewska et al., 1971) of phage induction with UV light from strains that were classified as avian or 'atypical' mycobacteria, but evidence of strain characterization was not presented and these phages were able to lyse mainly saprophytic mycobacteria or occasionally $\boldsymbol{M}$. tuberculosis.

It was somewhat surprising to find that phage induced from strain $6 / 8$ by treatment with mitomycin C and single-plaque purified on $M$. smegmatis was capable of lysing strain $6 / 8$. This could indicate that a subculture of this strain lost the temperate phage or that this strain is pseudolysogenic with a majority of the cells being sensitive to the phage. The molecular nature of the interaction of phage TM4 with its host cell is unclear but it probably represents a form of pseudolysogeny. A similar situation has been reported with a spontaneously released phage from a strain of $M$. bovis (Tageldin et al., 1981). In a Bacillus stearothermophilus strain mitomycin C enhanced the release of a pseudolysogenic phage $\phi \mathrm{u}-4$, which could be detected by plaque formation on the strain from which it was induced (Holmes et al., 1981). 
The phages we have induced from members of the MAIS complex should prove useful in understanding the genetics of this clinically important but genetically poorly characterized group of mycobacteria.

This research was supported by grant AI 18357 from the US-Japan Cooperative Medical Sciences Program of the National Institute of Allergy and Infectious Diseases, National Institutes of Health, US Department of Health, Education and Welfare.

\section{REFERENCES}

BAEss, I. (1970). Report on a pseudolysogenic mycobacterium and a review of the literature concerning pseudolysogeny. Acta pathologica et microbiologica scandinavica B79, 428-434.

Barrow, W. W., Ullom, B. P. \& Brennan, P. J. (1980). Peptidoglycolipid nature of the superficial cell wall sheath of smooth-colony-forming mycobacteria. Journal of Bacteriology 144, 814-822.

Bradley, D. E. \& RUTHERFORD, E. L. (1975). Basic characterization of a lipid containing bacteriophage specific for plasmids of the $\mathbf{P}, \mathbf{N}$, and $\mathrm{W}$ compatability groups. Canadian Journal of Microbiology 21, $152-163$.

Brennan, P. J. \& Goren, M. B. (1979). Structural studies on the type-specific antigens and lipids of the Mycobacterium avium/Mycobacterium intracellulare/ Mycobacterium scrofulaceum serocomplex. Journal of Biological Chemistry 254, 4205-4211.

Brennan, P. J., Mayer, H., Aspinali, G. O. \& Nam SHIN, J. E. (1981). Structures of the glycopeptidolipid antigens from serovars in the Mycobacterium avium/Mycobacterium intracellulare/Mycobacterium scrofulaceum serocomplex. European Journal of Biochemistry 115, 7-15.

Brennan, P. J., Heifets, M. J. \& Ullom, B. P. (1982). Thin layer chromatography of lipid antigens as means of identifying nontuberculous mycobacteria. Journal of Clinical Microbiology 15, 447-455.

BRUNNer, R. \& VinOGRAD, J. (1965). The evalution of standard sedimentation coefficients of sodium RNA and sodium DNA from sedimentation velocity data in concentrated $\mathrm{NaCl}$ and $\mathrm{CsCl}$ solutions. Biochimica et biophysica acta 108, 18-29.

BuraczewsKa, M., ManowsKa, W. \& RdultowsKa, H. (1971). Induction of phage from lysogenic mycobacteria by means of ultraviolet irradiation. American Review of Respiratory Diseases 104, 760762.

BuraczewsKa, M., KwiatKowski, B., ManowsKa, W. \& RDULTOWSKA, H. (1972). Ultrastructure of some mycobacteriophages. American Review of Respiratory Diseases 105, 22-29.

Cohn, M. L., Waggoner, R. F. \& McClatchy, J. K. (1968). The $7 \mathrm{H} 11$ medium for the cultivation of mycobacteria. American Review of Respiratory Diseases 98, 1064-1067.

Crawford, J. T., Fitzhugh, J. K. \& Bates, J. H. (1981). Phage typing of the Mycobacterium avium/intracellulare-scrofulaceum complex. American Review of Respiratory Diseases 124, 559-562.

DRAPER, P. (1974). The mycoside capsule of Mycobacterium avium 357. Journal of General Microbiology 83, $431-433$.
DRAPER, P. (1982). The physiology of mycobacteria. In The Biology" of the Mycobacteria, vol. I, pp. 9-52. Edited by C. Ratledge \& J. Stanford. London \& New York: Academic Press.

DRAPER, P. \& REES, R. J. W. (1973). The nature of the electron-transparent zone that surrounds Mycobacterium lepraemurium inside host cells. Journal of General Microbiology 77, 79-87.

Furuchi, A. \& ToKunaga, T. (1972). Nature of the receptor substance of Mycobacterium smegmatis for D4 bacteriophage adsorption. Journal of Bacteriology 14, 404-409.

Goren, M. B., McClatchy, J. K., Martens, B. \& BroKL, O. (1972). Mycosides C: behaviour as receptor site substance for mycobacteriophage D4. Journal of Virology 9, 999-1003.

Grange, J. M. (1982). The genetics of mycobacteria and mycobacteriophages. In The Biology of the Mycobacteria, vol. I, pp. 309-351. Edited by C. Ratledge \& J. Stanford. London \& New York: Academic Press.

Holmes, D., WoJtkiewicz, P. \& BarRidge, B. D. (1981). Properties of the virulent form of a mitomycin C- or temperature-induced thermophilic bacteriophage. Journal of General Virology 52, 141144.

ImAedA, T. \& Rieber, M. (1968). Mitomycin Cinduced phage-like particles in a mutant of Mycobacterium tuberculosis BCG. Journal of Bacteriology 96, 557-559.

JoNES, W. D. \& GREenBerg, J. (1978). Modification of methods used in bacteriophage typing of Mycobacterium tuberculosis isolates. Joumal of Clinical Microbiology 7, 467-469.

RADO, T. A. \& BATES, J. H. (1980). Mycobacteriophage structure and function: a review. Advances in Tuberculosis Research 20, 64-91.

Russell, R. L., JanN, G. J. \& Froman, S. (1960). Lysogeny in the mycobacteria. I. Establishment of lysogeny. American Review of Respiratory Diseases 82, 384-393.

Tageldin, M. H., El Hassan, A. M. \& Mustafa, I. E. (1981). Spontaneous release of mycophages from lysogenic bovine strains. Tubercule 62, 263-269.

TAKEYA, K. \& ToKIWA, H. (1972). Mycobacteriocin classification of rapidly growing mycobacteria. International Journal of Systematic Bacteriology 22, $178-180$.

Takeya, K., Yoshimura, T., Yamamura, K. \& Toda, T. (1959). Studies on the biologic properties of mycobacteriophage. American Review of Respiratory Diseases 80, 543-553.

TSANG, A. Y., DRUPA, I., GoldberG, M., 
McClatchy, J. K. \& Brennan, P. J. (1983). Use of serology and thin-layer chromatography for the assembly of an authenticated collection of serovars within the Mycobacterium avium-Mycobacterium intracellulare-Mycobacterium scrofulaceum complex. International Journal of Systematic Bacteriology 33, 285-292.
WolinSKY, E. (1979). Nontuberculous mycobacteria and associated diseases. American Review of Respiratory Diseases 119, 107-159.

Wolinsky, E. \& Schaefer, W. B. (1973). Proposed numbering scheme for mycobacterial serotypes by agglutination. International Journal of Systematic Bacteriology 23, 182-183. 